\title{
FREE ALGEBRAS IN DIVISION RINGS WITH AN INVOLUTION
}

\author{
VITOR O. FERREIRA, ÉRICA Z. FORNAROLI, AND JAIRO Z. GONÇALVES
}

\begin{abstract}
Some general criteria to produce explicit free algebras inside the division ring of fractions of skew polynomial rings are presented. These criteria are applied to some special cases of division rings with natural involutions, yielding, for instance, free subalgebras generated by symmetric elements both in the division ring of fractions of the group algebra of a torsion free nilpotent group and in the division ring of fractions of the first Weyl algebra.
\end{abstract}

\section{INTRODUCTION}

It has been conjectured by Makar-Limanov in [15] that a division ring which is infinite dimensional over its center $k$ and finitely generated (as a division algebra over $k$ ) must contain a noncommutative free $k$-subalgebra. Makar-Limanov himself provided evidence for this in [13, where it is proved that the division ring of fractions of the first Weyl algebra over the rational numbers contains a free subalgebra of rank 2, and in 14, where the case of the division ring of fractions of a group algebra of a torsion free nonabelian nilpotent groups is tackled. Various authors have dealt with this problem and Makar-Limanov's conjecture has been verified in many families of division rings (see, e.g., [12, 16, 9, 7, 18, 11, 19, 2, 10, 8, ,5, 3, 17, 4, 6, 1, ).

Division rings often come equipped with an involution. That is the case, for instance, of division rings of fractions of group algebras which are Ore domains. These have natural involutions induced by involutions on the group.

After the work in [10, it has become apparent that an involutional version of Makar-Limanov's conjecture should be investigated. To be more precise, given a field $k$ and a division $k$-algebra $D$, a $k$-linear map $*: D \rightarrow D$ satisfying $(a b)^{*}=b^{*} a^{*}$ and $a^{* *}=a$ for all $a, b \in D$ is called a $k$-involution. An element $a \in D$ is said to be symmetric with respect to the involution $*$ if $a^{*}=a$. Our aim in this paper is to contribute with supporting evidence to the following conjecture.

Conjecture 1.1. Let $D$ be a division ring with center $k$, and let $*$ be a $k$-involution on $D$. If $D$ is infinite dimensional over $k$ and finitely generated as a division $k$ algebra, then there exist two symmetric elements in $D$ which freely generate a free $k$-subalgebra of $D$.

Key words and phrases. Free associative algebras, field of fractions of group algebras, involutions, symmetric elements.

The first author was partially supported by Fapesp-Brazil, Proj. Temático 2009/52665-0.

The third author was supported by Grant CNPq 300.128/2008-8 and by Fapesp-Brazil, Proj. Temático 2009/52665-0. 
In [5], this conjecture has been proved to hold for the division ring of fractions, inside the division ring of Malcev-Neumann series, of the group algebra of a nonabelian orderable group $G$ with respect to an involution induced by the canonical (inverting) involution on $G$.

Here, we present proofs to the following two further special cases of Conjecture 1.1. which can be regarded as involutional versions of Makar-Limanov's early results.

Theorem 1.2. Let $D$ be the division ring of fractions of the group algebra $k \Gamma$ of the Heisenberg group $\Gamma$ over the field $k$ and let $*$ be a $k$-involution of $D$ which is induced from an involution on $\Gamma$. Then $D$ contains a free $k$-algebra of rank 2 freely generated by symmetric elements.

By the Heisenberg group, one understands the free nilpotent group of class 2 generated by 2 elements. It can be presented by

$$
\Gamma=\langle x, y:[[x, y], x]=[[x, y], y]=1\rangle,
$$

where $[g, h]$ denotes the commutator $g^{-1} h^{-1} g h$ of elements $g, h$ in a group.

Theorem 1.3. Let $A_{1}=\mathbb{Q}\langle s, t: s t-t s=1\rangle$ denote the first Weyl algebra over the field $\mathbb{Q}$ of rational numbers and let $*$ denote the $\mathbb{Q}$-involution of $A_{1}$ such that $s^{*}=-s$ and $t^{*}=t$. Then the division ring of fractions $D_{1}$ of $A_{1}$ contains a free $\mathbb{Q}$-subalgebra of rank 2 freely generated by symmetric elements with respect to the extension of $*$ to $D_{1}$.

Theorems 1.2 and 1.3 will follow from criteria that generalize the method developed by Bell and Rogalski in [2]. These will also provide simpler proofs of [18, Theorem A] and [19, Theorem 1]. As a special case, we obtain the following result.

Theorem 1.4. Let $F$ be a field, let $K=F\left(X_{1}, \ldots, X_{n}\right)$ be the rational function field in $n$ indeterminates over $F$, and let $\sigma$ be an $F$-automorphism of $K$ of infinite order that extends one from the polynomial algebra $F\left[X_{1}, \ldots, X_{n}\right]$. Then, the division algebra $K(X ; \sigma)$ contains a noncommutative free $F$-subalgebra.

\section{Free SUBALGEBRAs OF FIELDS OF FRACTIONS OF SKEW POLYNOMIAL RINGS}

In this section we offer generalizations of the method of [2] to construct free algebras inside division ring of fractions of skew polynomial rings.

Let $k$ be a field and let $D$ be a division $k$-algebra. Let $\sigma: D \rightarrow D$ be a $k$ automorphism and let $\delta: D \rightarrow D$ be a $\sigma$-derivation (that is, a $k$-linear map satisfying $\delta(\alpha \beta)=\sigma(\alpha) \delta(\beta)+\delta(\alpha) \beta$, for all $\alpha, \beta \in D)$. Denote by $D[X ; \sigma, \delta]$ the skew polynomial ring in the indeterminate $X$ such that $X \alpha=\sigma(\alpha) X+\delta(\alpha)$, for all $\alpha \in D$, and let $D(X ; \sigma, \delta)$ denote its division ring of fractions. Given $a_{0}, a_{1}, b_{0}, b_{1} \in k$, consider the polynomials $f=a_{0}+a_{1} X, g=b_{0}+b_{1} X \in k[X] \subseteq D[X ; \sigma, \delta]$. Also, let $\psi: D \rightarrow D$ be the map defined by $\psi=a_{1} \delta+a_{0}(\mathrm{Id}-\sigma)$, where Id stands for the identity map from $D$ to $D$. (Note that $\psi$ is again a $\sigma$-derivation.) Finally, let $E=\operatorname{ker} \psi$.

In what follows, we will further assume that $a_{1} \neq 0$ and that $\Xi=g f^{-1} \in$ $D(X ; \sigma, \delta) \backslash k$.

Under these hypotheses, we shall prove the following two theorems.

Theorem 2.1. Let $\alpha \in D$ be such that

- $\left\{1, \alpha, \alpha^{2}\right\}$ is left linearly independent over $\sigma(E)$ and 
- $\psi(D) \cap\left(\sigma(E)+\sigma(E) \alpha+\sigma(E) \alpha^{2}\right)=\{0\}$.

If either

(i) $b_{1}=0$ or

(ii) $b_{0}=0$ and $\delta=0$,

then the set $\{\alpha \Xi, \Xi \alpha\}$ freely generates a free $k$-subalgebra in $D(X ; \sigma, \delta)$.

Proof. Consider the set

$$
S=\left\{\left(i_{1}, \ldots, i_{t}\right): t \geq 1, i_{j} \in\{0,1,2\}, \text { for all } j \in\{1, \ldots, t\}\right\} .
$$

Given $I=\left(i_{1}, \ldots, i_{t}\right) \in S$, consider the elements in $D(X ; \sigma, \delta)$ defined by

$$
R_{I}=\alpha^{i_{1}} \Xi \alpha^{i_{2}} \Xi \ldots \alpha^{i_{t-1}} \Xi \alpha^{i_{t}} \Xi \alpha
$$

and

$$
L_{I}=\alpha^{i_{1}} \Xi \alpha^{i_{2}} \Xi \ldots \alpha^{i_{t-1}} \Xi \alpha^{i_{t}} \alpha \Xi .
$$

The set $\mathcal{B}=\{1\} \cup\left\{R_{I}: I \in S\right\} \cup\left\{L_{1}: I \in S\right\}$ (properly) contains all the words in the letters $\alpha \Xi$ and $\Xi \alpha$. Therefore, if we prove that $\mathcal{B}$ is linearly independent over $k$, we will have proved that $\alpha \Xi$ and $\Xi \alpha$ freely generate a free $k$-algebra.

In order to show that $\mathcal{B}$ is indeed linearly independent over $k$, we shall introduce new auxiliary elements. Given $I=\left(i_{1}, \ldots, i_{t}\right) \in S$, let

$$
V_{I}=\Xi \alpha^{i_{1}} \Xi \alpha^{i_{2}} \Xi \ldots \alpha^{i_{t-1}} \Xi \alpha^{i_{t}} \Xi \alpha,
$$

that is, $V_{I}=\Xi R_{I}$. We shall also define $V_{\varnothing}=\Xi$.

Given $I=\left(i_{1}, \ldots, i_{t}\right) \in S$, define the truncation of $I$ to be $I^{\prime}=\left(i_{2}, \ldots, i_{t}\right)$ if $t \geq 2$, and $I^{\prime}=\varnothing$ if $t=1$. So, in $D(X ; \sigma, \delta)$, the following relations hold:

$$
\Xi^{-1} V_{\varnothing}=1 \text { and } \Xi^{-1} V_{I}=R_{I}=\alpha^{i_{1}} V_{I^{\prime}}
$$

for all $I \in S$.

For $I=\left(i_{1}, \ldots, i_{t}\right) \in S$, we define the length of $I$ to be $\mu(I)=t$. Also, we set $\mu(\varnothing)=0$.

We claim that if $\left\{V_{I}: I \in S \cup\{\varnothing\}\right\}$ is left linearly independent over $D$, then $\mathcal{B}$ is linearly independent over $k$. Indeed, suppose $\left\{V_{I}: I \in S \cup\{\varnothing\}\right\}$ is left linearly independent over $D$ and that

$$
b+\sum_{I \in S} c_{I} R_{I}+\sum_{I \in S} d_{I} L_{I}=0
$$

is a linear combination of elements of $\mathcal{B}$ with coefficients $b, c_{I}, d_{I}$ from $k$ resulting in 0. Multiplying (2) by $\Xi \alpha$ on the right, one obtains a relation of the form

$$
\sum_{I \in S} e_{I} R_{I}=0
$$

with $e_{I} \in k$. Note that, by doing that, all of the elements $R_{I}$ in (3) are distinct. Hence, in view of (11), we get

$$
0=\sum_{I \in S} e_{I} R_{I}=\sum_{I \in S} e_{I} \alpha^{i_{1}} V_{I^{\prime}}
$$

For each $I=\left(i_{1}, \ldots, i_{t}\right) \in S$, there are exactly 3 elements in $S$ which have truncation $I^{\prime}$, they are

$$
I_{0}=\left(0, i_{1}, \ldots, i_{t}\right), \quad I_{1}=\left(1, i_{1}, \ldots, i_{t}\right) \quad \text { and } \quad I_{2}=\left(2, i_{1}, \ldots, i_{t}\right) .
$$


Thus, since $\left\{V_{I}: I \in S \cup\{\varnothing\}\right\}$ is left linearly independent over $D$, it follows that, for each $I \in S$, one has

$$
e_{I_{0}}+e_{I_{1}} \alpha+e_{I_{2}} \alpha^{2}=0 .
$$

But, by hypothesis, $\left\{1, \alpha, \alpha^{2}\right\}$ is linearly independent over $k$ (for $\sigma(E) \supseteq k$ ); therefore, $e_{I_{0}}=e_{I_{1}}=e_{I_{2}}=0$. This proves that all the coefficients in (3), which are the same as the ones in (2), are zero. So, $\mathcal{B}$ is linearly independent over $k$.

Our next task is to show that $\left\{V_{I}: I \in S \cup\{\varnothing\}\right\}$ is left linearly independent over $D$. We shall split the proof in two parts, depending on the conditions (i) or (ii) in the statement of the theorem.

First suppose that condition (i) holds, that is, that $b_{1}=0$. In this case, we must have $b_{0} \neq 0$. We shall show the stronger statement that $\left\{V_{I}: I \in S \cup\{\varnothing\}\right\}$ is left linearly independent over $D$ modulo the subspace $D[X ; \sigma, \delta]$. By contradiction, suppose there exists a relation

$$
\sum_{I \in S \cup\{\varnothing\}} \beta_{I} V_{I}=h \in D[X ; \sigma, \delta],
$$

with $\beta_{I} \in D$ not all zero. Among all those relations, choose one with $r=\max \{\mu(I)$ : $\left.\beta_{I} \neq 0\right\}$ minimal. Moreover, among those, choose one with the smallest number of nonzero coefficients $\beta_{I}$ for $I$ with $\mu(I)=r$. Note that $r \geq 1$, otherwise we would have $\Xi \in D[X ; \sigma, \delta]$, which is impossible. Clearly, we can further assume that our relation (4), beyond being minimal in the sense described above, has $\beta_{T}=1$ for some $T \in S$ with $\mu(T)=r$, by multiplying it by a nonzero element of $D$ on the left if necessary.

Recall that $\Xi=g f^{-1}=b_{0}\left(a_{0}+a_{1} X\right)^{-1}$. Hence, $\Xi^{-1}=\left(a_{0}+a_{1} X\right) b_{0}^{-1}$. It, then, follows from (1) that

$$
X V_{\varnothing}=-a_{1}^{-1} a_{0} V_{\varnothing}+a_{1}^{-1} b_{0} \quad \text { and } \quad X V_{I}=-a_{1}^{-1} a_{0} V_{I}+a_{1}^{-1} b_{0} \alpha^{i_{1}} V_{I^{\prime}},
$$

for all $I \in S$. Multiplying (4) by $X$ on the left, and using (5), yields

$$
\begin{aligned}
X h= & \sum_{I \in S \cup\{\varnothing\}} X \beta_{I} V_{I}=\sum_{I \in S \cup\{\varnothing\}}\left(\sigma\left(\beta_{I}\right) X+\delta\left(\beta_{I}\right)\right) V_{I} \\
= & \sigma\left(\beta_{\varnothing}\right) X V_{\varnothing}+\delta\left(\beta_{\varnothing}\right) V_{\varnothing}+\sum_{I \in S}\left(\sigma\left(\beta_{I}\right) X+\delta\left(\beta_{I}\right)\right) V_{I} \\
= & \sigma\left(\beta_{\varnothing}\right)\left(-a_{1}^{-1} a_{0} V_{\varnothing}+a_{1}^{-1} b_{0}\right)+\delta\left(\beta_{\varnothing}\right) V_{\varnothing} \\
& +\sum_{I \in S} \sigma\left(\beta_{I}\right)\left(-a_{1}^{-1} a_{0} V_{I}+a_{1}^{-1} b_{0} \alpha^{i_{1}} V_{I^{\prime}}\right)+\sum_{I \in S} \delta\left(\beta_{I}\right) V_{I} \\
= & \sum_{I \in S \cup\{\varnothing\}}\left(\delta\left(\beta_{I}\right)-a_{1}^{-1} a_{0} \sigma\left(\beta_{I}\right)\right) V_{I}+\sum_{I \in S} a_{1}^{-1} b_{0} \sigma\left(\beta_{I}\right) \alpha^{i_{1}} V_{I^{\prime}}+a_{1}^{-1} b_{0} \sigma\left(\beta_{\varnothing}\right) .
\end{aligned}
$$

Multiplying this by $a_{1}$ and summing with $a_{0} h$, one gets

$$
\begin{aligned}
f h= & \left(a_{0}+a_{1} X\right) h=a_{0} h+a_{1} X h=\sum_{I \in S \cup\{\varnothing\}} a_{0} \beta_{I} V_{I}+ \\
& +\sum_{I \in S \cup\{\varnothing\}}\left(a_{1} \delta\left(\beta_{I}\right)-a_{0} \sigma\left(\beta_{I}\right)\right) V_{I}+\sum_{I \in S} b_{0} \sigma\left(\beta_{I}\right) \alpha^{i_{1}} V_{I^{\prime}}+b_{0} \sigma\left(\beta_{\varnothing}\right) \\
= & \sum_{I \in S \cup\{\varnothing\}} \psi\left(\beta_{I}\right) V_{I}+\sum_{I \in S} b_{0} \sigma\left(\beta_{I}\right) \alpha^{i_{1}} V_{I^{\prime}}+b_{0} \sigma\left(\beta_{\varnothing}\right) .
\end{aligned}
$$


Therefore, one has

$$
\sum_{I \in S \cup\{\varnothing\}} \psi\left(\beta_{I}\right) V_{I}+\sum_{I \in S} b_{0} \sigma\left(\beta_{I}\right) \alpha^{i_{1}} V_{I^{\prime}}=f h-b_{0} \sigma\left(\beta_{0}\right) \in D[X ; \sigma, \delta] .
$$

The coefficient of $V_{T}$ in (6) is $\psi\left(\beta_{T}\right)=\psi(1)=0$. Moreover, no new nonzero coefficient of a $V_{I}$ with $\mu(I)=r$ appears in (6). By the minimality of (4), all the coefficients of the $V_{I}$ in (6) are zero. If $\mu(I)=r$, the coefficient of $V_{I}$ in (6) is $\psi\left(\beta_{I}\right)$, so, in particular, it follows that $\beta_{I} \in E=\operatorname{ker} \psi$ for all $I \in S$ with $\mu(I)=r$. Now, there are exactly 3 elements $I_{0}, I_{1}, I_{2}$ in $S$ whose truncations equal $T^{\prime}$. Since all three have length $r$, if follows that $\beta_{I_{0}}, \beta_{I_{1}}, \beta_{I_{2}} \in E$. But the coefficient of $V_{T^{\prime}}$ in (6) is $\psi\left(\beta_{T^{\prime}}\right)+b_{0} \sigma\left(\beta_{I_{0}}\right)+b_{0} \sigma\left(\beta_{I_{1}}\right) \alpha+b_{0} \sigma\left(\beta_{I_{2}}\right) \alpha^{2}$. So,

$$
\psi\left(\beta_{T^{\prime}}\right)=\sigma\left(-b_{0} \beta_{I_{0}}\right)+\sigma\left(-b_{0} \beta_{I_{1}}\right) \alpha+\sigma\left(-b_{0} \beta_{I_{2}}\right) \alpha^{2},
$$

which is an element of $\psi(D) \cap\left(\sigma(E)+\sigma(E) \alpha+\sigma(E) \alpha^{2}\right)=\{0\}$. Since $\left\{1, \alpha, \alpha^{2}\right\}$ is left linearly independent over $\sigma(E)$, it follows that $\beta_{I_{0}}=\beta_{I_{1}}=\beta_{I_{2}}=0$. But $T \in\left\{I_{0}, I_{1}, I_{2}\right\}$. This contradicts the fact that $\beta_{T}=1$.

Now suppose that condition (ii) holds, that is, that $b_{0}=0$ and $\delta=0$. In this case, we must have $b_{1} \neq 0$ and $a_{0} \neq 0$. We shall show the stronger statement that $\left\{V_{I}: I \in S \cup\{\varnothing\}\right\}$ is left linearly independent over $D$ modulo the subspace $D\left[X, X^{-1} ; \sigma\right]$. By contradiction, suppose there exists a relation

$$
\sum_{I \in S \cup\{\varnothing\}} \beta_{I} V_{I}=h \in D\left[X, X^{-1} ; \sigma\right],
$$

with $\beta_{I} \in D$ not all zero. Among all those relations, choose one with $r=\max \{\mu(I)$ : $\left.\beta_{I} \neq 0\right\}$ minimal. Moreover, among those, choose one with the smallest number of nonzero coefficients $\beta_{I}$ for $I$ with $\mu(I)=r$. Note that $r \geq 1$, otherwise we would have $\Xi \in D\left[X, X^{-1} ; \sigma\right]$, which is impossible (for $a_{0} \neq 0$ ). Clearly, we can further assume that our relation (4), beyond being minimal in the sense described above, has $\beta_{T}=1$ for some $T \in S$ with $\mu(T)=r$, by multiplying it by a nonzero element of $D$ on the left if necessary.

It follows from (1) that

$$
X^{-1} V_{\varnothing}=-a_{1} a_{0}^{-1} V_{\varnothing}+b_{1} a_{0}^{-1} \quad \text { and } \quad X^{-1} V_{I}=-a_{1} a_{0}^{-1} V_{I}+b_{1} a_{0}^{-1} \alpha^{i_{1}} V_{I^{\prime}},
$$

for all $I \in S$. If one multiplies (7) by $X^{-1}$ on the left, relations (8) allow us to conclude that

$$
X^{-1} h=\sum_{I \in S \cup\{\varnothing\}}-a_{1} a_{0}^{-1} \sigma^{-1}\left(\beta_{I}\right) V_{I}+\sum_{I \in S} b_{1} a_{0}^{-1} \sigma^{-1}\left(\beta_{I}\right) \alpha^{i_{1}} V_{I^{\prime}}+b_{1} a_{0}^{-1} \sigma^{-1}\left(\beta_{\varnothing}\right) .
$$

This multiplied by $a_{1}^{-1} a_{0}^{2}$ and, then, summed with $-a_{0} h$ yields

$$
\begin{aligned}
& \sum_{I \in S \cup\{\varnothing\}} \psi\left(\sigma^{-1}\left(\beta_{I}\right)\right) V_{I}-\sum_{I \in S} b_{1} a_{1}^{-1} a_{0} \sigma^{-1}\left(\beta_{I}\right) \alpha^{i_{1}} V_{I^{\prime}} \\
& =-\left(a_{1}^{-1} a_{0}^{2} X^{-1}+a_{0}\right) h+b_{1} a_{1}^{-1} a_{0} \sigma^{-1}\left(\beta_{\varnothing}\right) \in D\left[X, X^{-1} ; \sigma\right] .
\end{aligned}
$$

The coefficient of $V_{T}$ in (9) is $\psi\left(\sigma^{-1}\left(\beta_{T}\right)\right)=\psi(1)=0$. By minimality, all the coefficients on the left-hand side of (9) are zero. In particular, if $\mu(I)=r$, the coefficient of $V_{I}$ is $0=\psi\left(\sigma^{-1}\left(\beta_{I}\right)\right)$. So, for $I$ with $\mu(I)=r$, one has $\beta_{I} \in \sigma(E)=E$. (This last equality follows from the fact that, in this case, $E=\operatorname{ker}(\operatorname{Id}-\sigma)$; so $\sigma(E)=E$.) The rest of the argument is analogous to the one in the first case. 
Theorem 2.2. Let $n$ be an integer with $n \geq 2$. Let $\alpha_{1}, \ldots, \alpha_{n} \in D$ be such that

- $\left\{\alpha_{1}, \ldots, \alpha_{n}\right\}$ is left linearly independent over $\sigma(E)$ and

- $\psi(D) \cap\left(\sigma(E) \alpha_{1}+\cdots+\sigma(E) \alpha_{n}\right)=\{0\}$.

If either

(i) $b_{1}=0$ or

(ii) $b_{0}=0$ and $\delta=0$,

then the set $\left\{\alpha_{1} \Xi, \ldots, \alpha_{n} \Xi\right\}$ freely generates a free $k$-subalgebra in $D(X ; \sigma, \delta)$.

Proof. (Sketch.) We consider the set

$$
\begin{aligned}
& S=\left\{\left(\left(i_{1}\right), \ldots,\left(i_{t}\right)\right): t \geq 1,\left(i_{j}\right)=\left(i_{j 1}, \ldots, i_{j n}\right), i_{j l} \in\{0,1\},\right. \\
& \left.\qquad \sum_{l=1}^{n} i_{j l}=1, \text { for all } j=1, \ldots, t\right\} .
\end{aligned}
$$

Given $I=\left(\left(i_{1}\right), \ldots,\left(i_{t}\right)\right) \in S$, one defines

$$
W_{I}=\alpha_{1}^{i_{11}} \ldots \alpha_{n}^{i_{1 n}} \Xi \alpha_{1}^{i_{21}} \ldots \alpha_{n}^{i_{2 n}} \Xi \ldots \alpha_{1}^{i_{t 1}} \ldots \alpha_{n}^{i_{t n}} \Xi .
$$

The set of all nonempty words in the letters $\alpha_{1} \Xi, \ldots, \alpha_{n} \Xi$ coincides with $\left\{W_{I}: I \in\right.$ $S\}$. Our task is, thus, to show that $\mathcal{B}=\{1\} \cup\left\{W_{I}: I \in S\right\}$ is linearly independent over $k$.

Here, for $I=\left(\left(i_{1}\right), \ldots,\left(i_{t}\right)\right) \in S$, its length is defined to be $t$ and its truncation $I^{\prime}=\left(\left(i_{2}\right), \ldots,\left(i_{t}\right)\right) \in S$, if $t \geq 2$. If $I$ has length 1 , its truncation is defined to be $I^{\prime}=\varnothing$. It follows from the definition of $S$ that given $I \in S$, there exist exactly $n$ elements of $S$, all of them with the same length as $I$, having truncation $I^{\prime}$ (clearly, one of them is $I$ itself).

Defining $V_{I}=\Xi W_{I}$, for $I \in S$, and $V_{\varnothing}=\Xi$, one can show, following the lines of the proof of Theorem 2.1 above, that, first, if $\left\{V_{I}: I \in S \cup\{\varnothing\}\right\}$ is left linearly independent over $D$, then $\mathcal{B}$ is linearly independent over $k$. Moreover, the proof, in Theorem 2.1, that $\left\{V_{I}: I \in S \cup\{\varnothing\}\right\}$ is left linearly independent over $D$, under both condition (i) or condition (ii), can also be adapted to the present context.

Remark 2.3. Setting $\sigma$ to be the identity automorphism of $D$, Theorem 2.2 can be used to recover both [18, Theorem A] and Makar-Limanov's result of [13], producing free subalgebras inside the division ring of fractions of the first Weyl algebra over the rationals. Indeed, if $D_{1}$ denotes the division ring of fractions of the first Weyl algebra $A_{1}=\mathbb{Q}\langle s, t: s t-t s=1\rangle$, then, via the identification $s \mapsto X, D_{1}$ coincides with the division ring of fractions $\mathbb{Q}(t)(X ; \delta)$ of the skew polynomial ring $\mathbb{Q}(t)[X ; \delta]$, where $\delta$ is the usual derivation on the rational function field $\mathbb{Q}(t)$, that is, the one satisfying $\delta(t)=1$. Here, the rational functions $\alpha_{1}=\frac{1}{t}$ and $\alpha_{2}=\frac{1}{t(1-t)}$ satisfy the hypotheses of Theorem 2.2 hence, taking $a_{0}=b_{0}=0$ and $a_{1}=b_{1}=1$, it follows that $\alpha_{1} X^{-1}$ and $\alpha_{2} X^{-1}$ generate a free $\mathbb{Q}$-subalgebra in $\mathbb{Q}(t)(X ; \delta)$, or, in other words, $(s t)^{-1}$ and $(1-t)^{-1}(s t)^{-1}$ generate a free $\mathbb{Q}$-subalgebra of $D_{1}$.

Observe that Theorem 2.2 recovers Makar-Limanov's result, which does not occur with Theorem 2.2 in [2], as pointed out by Bell and Rogalski.

In Section 4, we shall see that Theorem 2.2 can also provide a pair of symmetric elements of $D_{1}$ generating a free algebra, with respect to a natural involution on $D_{1}$. 


\section{Free symmetric subalgebras AND the Heisenberg Group}

Let $k$ be a field, let $\Gamma=\langle x, y:[[x, y], x]=[[x, y], y]=1\rangle$ be the Heisenberg group and let $*$ be an involution on $\Gamma$. Then $*$ can be linearly extended to a $k$-involution * on the group algebra $k \Gamma$, which, in turn, has a unique extension to a $k$-involution on the Ore division ring of fractions $D$ of the noetherian domain $k \Gamma$.

In this section, we shall present a proof of Theorem 1.2 , exhibiting two elements in $D$ which freely generate a free $k$-subalgebra and which are symmetric with respect to $*$. For that purpose, we shall make use of Theorem 2.1 and of the classification of involutions on $\Gamma$ given in 4 .

Recall that the center of $\Gamma$ is infinite cyclic, generated by $\lambda=[x, y]$. The attribution $\lambda \mapsto t, y \mapsto Y, x \mapsto X$ establishes a $k$-isomorphism between $D$ and the division ring $k((t)(Y))(X ; \sigma)$, where $k(t)$ stands for the field of rational functions in the indeterminate $t$ over $k, k(t)(Y)$ for the field of rational functions in the indeterminate $Y$ over $k(t)$, and $\sigma$ is the $k(t)$-automorphism of $k(t)(Y)$ satisfying $\sigma(Y)=t Y$.

Theorem 1.2 will follow from Theorem 2.1. after a judicious choice of elements $\alpha$ and $\Xi$. But, in order to verify the hypotheses of Theorem 2.1 in this setting, we shall need the following fact on automorphisms of rational function fields, whose proof is similar to the proof of [5, Lemma 1.4].

Lemma 3.1. Let $F$ be a field, let $t \in F \backslash\{0\}$ be an element which is not a root of unity, and let $\sigma$ be the $F$-automorphism of the rational function field $F(Y)$ such that $\sigma(Y)=t Y$. Let $\alpha \in F(Y) \backslash F[Y]$ be a rational function which has a unique pole and this pole is nonzero, and let $m$ be a positive integer. If $\beta \in F(Y)$ satisfies

$$
\sigma(\beta)-\beta \in F+F \alpha+\cdots+F \alpha^{m}
$$

then $\beta \in F$.

3.1. Proof of Theorem 1.2. As we have seen above, we can identify $D$ with $(k(t)(Y))(X ; \sigma)$. Taking $F=k(t)$ in Lemma 3.1 one sees that any rational function $\alpha \in F(Y)$ which has a unique pole and this pole is nonzero will satisfy the hypotheses of Theorem 2.1, therefore providing a pair $\left\{\alpha X(1-X)^{-1}, X(1-X)^{-1} \alpha\right\}$ inside $D$ which freely generates a free $k$-subalgebra. Now, according to [4, Theorem 3.4], up to equivalence, a $k$-involution $*$ on $D$ which is induced by an involution on $\Gamma$ must satisfy one of the following conditions:

(I) $X^{*}=\zeta X, Y^{*}=\eta Y$;

(II) $X^{*}=X^{-1}, Y^{*}=Y^{-1}$;

(III) $X^{*}=X, Y^{*}=\zeta Y^{-1}$;

(IV) $X^{*}=\zeta Y, Y^{*}=\zeta^{-1} X$;

the elements $\zeta$ and $\eta$ being powers of $t$ (and, therefore, central). In the first two cases, one has $t^{*}=t^{-1}$, and in the last two, $t$ is symmetric.

We shall treat each of the four types (I) (IV) separately.

(I) In this case, taking $\alpha=(1-Y)^{-1}$, we obtain elements $A=(1-Y)^{-1} X(1-$ $X)^{-1}$ and $B=X(1-X)^{-1}(1-Y)^{-1}$ freely generating a free subalgebra of $D$. Now consider the $k(t)$-automorphism $\psi$ of $D$ such that $\psi(Y)=(1+\eta) Y$ and $\psi(X)=(1+\zeta) X$. Since $(1+\eta) Y=Y+Y^{*}$ and $(1+\zeta) X=X+X^{*}$, it follows that $\psi(Y)$ and $\psi(X)$ are symmetric with respect to $*$. Thus, $\psi(A)^{*}=\psi(B)$. This implies that $\psi(A B)$ and $\psi(B A)$ are symmetric and, because $A B$ and $B A$ freely generate a free subalgebra of $D$, so do they.

(II) This is contained in Theorem 1.1 of [5]. 
(III) The rational function $\gamma=Z(\zeta-Z)^{-2}$ in the indeterminate $Z$ over the field $F=k(t)$ satisfies the conditions of Lemma 3.1 with respect to the automorphism $\tau$ such that $\tau(Z)=t^{2} Z$. Therefore, by Theorem 2.1, $\gamma X(1-X)^{-1}$ and $X(1-X)^{-1} \gamma$ freely generate a free $k$-subalgebra in $(k(t)(Z))(X ; \tau)$. Since the map $Z \mapsto Y^{2}$ establishes an isomorphism between $(k(t)(Z))(X ; \tau)$ and the subalgebra $\left(k(t)\left(Y^{2}\right)\right)(X ; \sigma)$ of $D$, it follows that, setting $\alpha=$ $Y^{2}\left(\zeta-Y^{2}\right)^{-2}$, the elements $A=Y^{2}\left(\zeta-Y^{2}\right)^{-2} X(1-X)^{-1}$ and $B=$ $X(1-X)^{-1} Y^{2}\left(\zeta-Y^{2}\right)^{-2}$ freely generate a free $k$-subalgebra of $D$. Since $A^{*}=B$, it follows that $A B$ and $B A$ form a pair of symmetric elements which freely generate a free subalgebra of $D$.

(IV) Here, taking $\alpha=Y(1-Y)^{-1}$, one gets the free pair $A=Y(1-Y)^{-1} X(1-$ $X)^{-1}$ and $B=X(1-X)^{-1} Y(1-Y)^{-1}$. If $\psi$ denotes the $k(t)$-automorphism of $D$ such that $\psi(X)=X$ and $\psi(Y)=\zeta Y$, it follows that $\{\psi(A), \psi(B)\}$ is a pair of symmetric elements which freely generates a free algebra in $D$.

\section{Free symmetric subalgebras and the first Weyl Algebra}

As we have seen in Remark 2.3. we can regard the division ring of fractions $D_{1}$ of the first Weyl algebra over $\mathbb{Q}$ as $\mathbb{Q}(t)(X ; \delta)$, where $\delta$ stands for the usual derivation on the rational function field $\mathbb{Q}(t)$.

In the proof of Theorem 1.3, we shall need the following consequence of Theorem 2.2

Corollary 4.1. Let $a, b \in \mathbb{Q}(t)$ be rational functions satisfying the following conditions:

- $\left\{a^{2}, a b\right\}$ is a $\mathbb{Q}$-linearly independent subset of $\mathbb{Q}(t)$, and

- $\delta(\mathbb{Q}(t)) \cap\left(\mathbb{Q} a^{2}+\mathbb{Q} a b\right)=\{0\}$.

Then, $a X^{-1} a$ and $b X^{-1} a$ freely generate a free $\mathbb{Q}$-subalgebra of $\mathbb{Q}(t)(X ; \delta)$.

Proof. By Theorem 2.2 the elements $a^{2} X^{-1}$ and $a b X^{-1}$ freely generate a free $\mathbb{Q}$ subalgebra of $\mathbb{Q}(t)(X ; \delta)$. Now, consider the set of monomials on the letters $A$ and $B$, and given $I=\left(i_{1}, j_{1}, i_{2}, j_{2}, \ldots, i_{n}, j_{n}\right)$ with $i_{k}, j_{k}$ nonnegative integers, let $M_{I}(A, B)$ be the monomial defined by

$$
M_{I}(A, B)=A^{i_{1}} B^{j_{1}} A^{i_{2}} B^{j_{2}} \ldots A^{i_{n}} B^{j_{n}} .
$$

Then, for any $I$, we have that

$$
a M_{I}\left(a X^{-1} a, b X^{-1} a\right) X^{-1}=M_{I}\left(a^{2} X^{-1}, a b X^{-1}\right) a X^{-1} .
$$

Hence, if $c_{I} \in \mathbb{Q}$ are such that only a finite number of them are nonzero and $\sum_{I} c_{I} M_{I}\left(a X^{-1} a, b X^{-1} a\right)=0$, multiplying this relation by $a$ on the left and by $X^{-1}$ on the right, we get, using (10),

$$
0=a\left(\sum_{I} c_{I} M_{I}\left(a X^{-1} a, b X^{-1} a\right)\right) X^{-1}=\left(\sum_{I} c_{I} M_{I}\left(a^{2} X^{-1}, a b X^{-1}\right)\right) a X^{-1}
$$

Since the set $\left\{a^{2} X^{-1}, a b X^{-1}\right\}$ is free, it follows that all the $c_{I}$ are zero. Therefore, $\left\{a X^{-1} a, b X^{-1} a\right\}$ is also free. 
4.1. Proof of Theorem 1.3. Consider the rational functions

$$
a=\frac{t}{1+t^{2}} \quad \text { and } \quad b=\frac{1}{1+t}
$$

in $\mathbb{Q}(t)$. Considering them as real functions in the variable $t$, we have

$$
\int\left(\frac{t}{1+t^{2}}\right)^{2} d t=\frac{1}{2}\left(\arctan t-\frac{t}{1+t^{2}}\right)+\text { constant }
$$

and

$$
\int\left(\frac{t}{1+t^{2}}\right)\left(\frac{1}{1+t}\right) d t=\frac{1}{4}\left(\ln \left(1+t^{2}\right)+2 \arctan t-2 \ln (1+t)\right)+\text { constant. }
$$

Developing $\arctan t, \ln \left(1+t^{2}\right)$ and $\ln (1+t)$ as power series in the interval $(0,1)$, we can easily check that $a$ and $b$ satisfy the conditions in Corollary 4.1. It follows that $\alpha=a s^{-1} a$ and $\beta=b s^{-1} a$ freely generate a free $\mathbb{Q}$-subalgebra of $D_{1}$. Hence, the symmetric elements $\alpha^{2}$ and $\alpha \beta$ also generate a free $\mathbb{Q}$-subalgebra of $D_{1}$.

\section{Free subalgebras in $k\left(X_{1}, \ldots, X_{n}\right)(X ; \sigma)$}

In this section we follow closely the arguments in [19, Section 4] and show that part of the proof of [19, Theorem 1] can be greatly simplified using Theorem 2.2 .

We start with a more general setting. Let $k$ be a field and let $R$ be a commutative $k$-algebra which is a factorial domain with group of units $k^{\dagger}=k \backslash\{0\}$. Let $\sigma$ be a nonidentity $k$-automorphism of $R$ and assume the the fixed ring of $R$ under $\sigma$ coincides with $k$. Extend $\sigma$ to the field of fractions $K$ of $R$. Theorem 1.4 will follow from the next result, in the statement of which, for $a \in k^{\dagger}$, we use the notation $R_{a}=\{r \in R: \sigma(r)=a r\}$.

Proposition 5.1. Under the above hypotheses, the division algebra $K(X ; \sigma)$ contains a noncommutative free $k$-subalgebra. More precisely, one of the following alternative possibilities must hold.

(i) Either $R_{a}=\{0\}$, for all $a \in k^{\dagger} \backslash\{1\}$. In this case, given any $\alpha \in K \backslash R$ whose denominator is a prime power, for any positive integer $m$, the set

$$
\left\{\alpha X(1-X)^{-1}, \alpha^{2} X(1-X)^{-1}, \ldots, \alpha^{m} X(1-X)^{-1}\right\}
$$

freely generates a free $k$-subalgebra in $K(X ; \sigma)$.

(ii) $\operatorname{Or} R \supseteq k[t]$, where $t$ is algebraically independent over $k$ and $\sigma$ satisfies $\sigma(t)=\lambda t$, for some $\lambda \in k$ which is not a root of unity. In this case, given any $b \in k$, for any positive integer $m$, the set

$$
\left\{(t-b)^{-1} X(1-X)^{-1},(t-b)^{-2} X(1-X)^{-1}, \ldots,(t-b)^{-m} X(1-X)^{-1}\right\}
$$

freely generates a free $k$-subalgebra in $K(X ; \sigma)$.

Proof. In case (i), take $\alpha \in K \backslash R$. By [19, Lemma 5], the set $\{1\} \cup\left\{\sigma^{j}\left(\alpha^{i}\right): i \geq\right.$ $1, j \geq 0\}$ is $k$-linearly independent. Moreover, if the denominator of $\alpha$ is a prime power, then, by [19, Lemma 7], the equation

$$
\sigma(\beta)-\beta=\sum_{i \geq 1} b_{i} \alpha^{i}
$$

has no solution with $b_{i} \in k$ and $\beta \in K \backslash k$. It follows from Theorem 2.2 that $\alpha X(1-X)^{-1}, \ldots, \alpha^{m} X(1-X)^{-1}$ freely generate a free $k$-algebra in $K(X ; \sigma)$ for any positive $m$. 
Now suppose that (i) does not hold, that is, there exists $\lambda \in k^{\dagger} \backslash\{1\}$ such that $R_{\lambda} \neq\{0\}$. By [19, Lemma 2], $\lambda$ is not a root of unity. Choose $t \in R_{\lambda} \backslash\{0\}$. Then, $\sigma(t)=\lambda t$ and we have an embedding $k(t)(X ; \sigma) \subseteq K(X ; \sigma)$. It follows from Lemma 3.1 and Theorem 2.2 that, for any $b \in k$ and any positive integer $m$, $(t-b)^{-1} X(1-X)^{-1},(t-b)^{-2} X(1-X)^{-1}, \ldots,(t-b)^{-m} X(1-X)^{-1}$ freely generate a free $k$-subalgebra in $k(t)(X ; \sigma)$ and, hence, in $K(X ; \sigma)$.

5.1. Proof of Theorem 1.4. The same argument used in the proof of [19, Corollary 2] holds. Let $M$ be the fixed subring of $S=k\left[X_{1}, \ldots, X_{n}\right]$ under the action of $\sigma$, let $R=S(M \backslash\{0\})^{-1}$, and let $k=M(M \backslash\{0\})^{-1}$. By Proposition [5.1] $K(X ; \sigma)$ contain a free $k$-subalgebra and, thus, by [16, Lemma 1], contains a free $F$-subalgebra.

\section{REFERENCES}

[1] Jason P. Bell and Jairo Z. Gonçalves, Free algebras and free groups in Ore extensions and free group algebras in division rings, J. Algebra 455 (2016), 235-250.

[2] Jason P. Bell and Daniel Rogalski, Free subalgebras of quotient rings of Ore extensions, Algebra Number Theory 6 (2012), 1349-1367.

[3] Jason P. Bell and Daniel Rogalski, Free subalgebras of division algebras over uncountable fields, Math. Z. 277 (2014), 591-609.

[4] Vitor O. Ferreira and Jairo Z. Gonçalves, Free symmetric and unitary pairs in division rings infinite-dimensional over their centers, Israel J. Math. 210 (2015), 297-321.

[5] Vitor O. Ferreira, Jairo Z. Gonçalves and Javier Sánchez, Free symmetric group algebras in division rings generated by poly-orderable groups, J. Algebra 392 (2013), 69-84.

[6] Vitor O. Ferreira, Jairo Z. Gonçalves and Javier Sánchez, Free symmetric algebras in division rings generated by enveloping algebras of Lie algebras, Internat. J. Algebra Comput. 25 (2015), 1075-1106.

[7] Leila M. Figueiredo, Jairo Z. Gonçalves and Mazi Shirvani, Free group algebras in certain division rings, J. Algebra 185 (1996), 298-313.

[8] Jairo Z. Gonçalves and Eduardo Tengan, Free group algebras in division rings, Internat. J. Algebra Comput. 22 (2012), no. 5, 1250044, 9 pp.

[9] Jairo Z. Gonçalves and Mazi Shirvani, On free group algebras in division rings with uncountable center, Proc. Amer. Math. Soc. 124 (1996), 685-687.

[10] Jairo Z. Gonçalves and Mazi Shirvani, A survey on free objects in division rings and in division rings with an involution, Comm. Algebra 40 (2012), 1704-1723.

[11] Alexander I. Lichtman, Free subalgebras in division rings generated by universal enveloping algebras, Algebra Colloq. 6 (1999), 145-153.

[12] Martin Lorenz, On free subalgebras of certain division algebras, Proc. Amer. Math. Soc. 98 (1986), 401-405.

[13] Leonid Makar-Limanov, The skew field of fractions of the Weyl algebra contains a free noncommutative subalgebra, Comm. Algebra 11 (1983), 2003-2006.

[14] Leonid Makar-Limanov, On group rings of nilpotent groups, Israel J. Math. 48 (1984), 244248.

[15] Leonid Makar-Limanov, On free subobjects of skew fields Methods in ring theory (Antwerp, 1983), 281-285, NATO Adv. Sci. Inst. Ser. C Math. Phys. Sci., 129, Reidel, Dordrecht, 1984.

[16] Leonid Makar-Limanov and Peter Malcolmson, Free subalgebras of enveloping fields, Proc. Amer. Math. Soc 111 (1991), 315-322.

[17] Javier Sánchez, Free group algebras in Malcev-Neumann skew fields of fractions, Forum Math. 26 (2014), 443-466.

[18] M. Shirvani and Jairo Z. Gonçalves, Free group algebras in the field of fractions of differential polynomial rings and enveloping algebras, J. Algebra 204 (1998), 372-385.

[19] Mazi Shirvani and Jairo Z. Gonçalves, Large free algebras in the ring of fractions of skew polynomial rings, J. London Math. Soc. (2) 60 (1999), 481-489. 
Department of Mathematics, University of São Paulo, São Paulo, 05508-090, Brazil

E-mail address: vofer@ime.usp.br

Department of Mathematics, Universidade Estadual de Maringá, Paraná, 87020-900, BRAZIL

E-mail address: ezancanella@uem.br

Department of Mathematics, University of São Paulo, São Paulo, 05508-090, Brazil

E-mail address: jz.goncalves@usp.br 